\title{
CHAIN TRANSFORMS
}

\section{CHARLES FOX}

1. Introduction. Iterations of the Laplace transform are well known and are of the form $[1$, p. 325]:

$$
\begin{aligned}
& h_{2}(x)=\int_{0}^{\infty} e^{-x u} h_{1}(u) d u, \\
& h_{3}(x)=\int_{0}^{\infty} e^{-x u} h_{2}(u) d u, \\
& \int_{0}^{\infty} \frac{h_{1}(u)}{x+u} d u=h_{3}(x) .
\end{aligned}
$$

We shall refer to this system as a chain transform of order 3 . The main purpose of this paper is to give a general theory of chain transforms of all orders and the results obtained will include as special cases the generalized theory of Fourier transforms [2, Chap. 8], which are of order 2, and also the theory given in my previous paper on chain transforms [3].

2. Definition of chain transforms of order $n$. Consider an assembly of $n$ equations consisting of two sub-assemblies, one of $i$ and the other of $j$ equations, where $i+j=n, i, j, n$ being positive integers. These sub-assemblies are of the form:

$$
\begin{gathered}
g_{p+1}(x)=\int_{0}^{\infty} r_{p}\left(\frac{x}{u}\right) g_{p}(u) \frac{d u}{u}, \\
\int_{0}^{\infty} l_{q}\left(\frac{x}{u}\right) g_{q+1}(u) \frac{d u}{u}=g_{q}(x),
\end{gathered}
$$

where $p$ runs through $i$ integers and $q$ through $j$ integers, not necessarily in consecutive fashion. Between them $p$ and $q$ run through the first $n$ positive integers. We shall refer to the functions $r_{p}(x)$ as kernels on the right, or more briefly as $r$-kernels, and the functions $l_{q}(x)$ as kernels on the left or as $l$-kernels. Since each equation contains a kernel, there are $n$ of them altogether. They may all differ from each other or some may be equal.

Since $p$ and $q$, between them, run through the first $n$ integers the functions $g_{i}(x)$ commence with $g_{1}(x)$ and terminate with $g_{n+1}(x)$.

Received by the editors December 14, 1953. 
Two conditions are required before the system (4), (5) can be called a chain transform of order $n$. They are:

(i) Each of the functions $g_{2}(x), g_{3}(x), \cdots, g_{n}(x)$ must occur twice in (4), (5), once on the left-hand side and once on the right. This leaves one place on the left and one on the right to be occupied by $g_{1}(x)$ and $g_{n+1}(x)$.

(ii) Also

$$
g_{n+1}(x)=g_{1}(x) .
$$

In this case each function $g_{i}(x), i=1,2, \cdots, n$, occurs twice in (4), (5), once on the left-hand side and once on the right.

For simplicity we shall assume that no two of the functions $g_{i}(x)$, $i=1,2, \cdots, n$, are equal, although this is not essential.

In order to prove that the system (4), (5) is a chain transform of order $n$ we must first know all the kernels and one of the $g_{i}(x)$ functions, $g_{1}(x)$ say. The equations then successively define the functions $g_{2}(x), g_{3}(x), \cdots, g_{n+1}(x)$ and the proof is completed by establishing the truth of (6).

This definition is much wider than the one given in my previous paper on chain transforms [3]. It specializes to the previous definition when all the kernels are either $r$-kernels or $l$-kernels.

To illustrate the definition consider equations (1), (2), and (3). In (1) replace $x$ by $1 / x$, in (2) replace $u$ by $1 / u$ and write $h_{1}(u)=g_{1}(u)$; $h_{2}(1 / x)=x g_{2}(x) ; h_{3}(x)=g_{3}(x)$. The equations then take the form

$$
\begin{aligned}
& g_{2}(x)=\int_{0}^{\infty}\left(\frac{u}{x} e^{-u / x}\right) g_{1}(u) \frac{d u}{u}, \\
& g_{3}(x)=\int_{0}^{\infty} e^{-x / u} g_{2}(u) \frac{d u}{u}, \\
& \int_{0}^{\infty} \frac{1}{(1+x / u)} g_{1}(u) \frac{d u}{u}=g_{3}(x) .
\end{aligned}
$$

This is evidently a chain transform of order 3 with two $r$-kernels and one $l$-kernel. It will appear later as a special case of Theorem 1 . It is worth noting that in the form (1), (2), (3) two of the kernels are equal but that in the form (7), (8), (9) this equality of the kernels is lost.

In the case $n=2$ with two $r$-kernels we have

$$
g_{2}(x)=\int_{0}^{\infty} r_{1}\left(\frac{x}{u}\right) g_{1}(u) \frac{d u}{u},
$$




$$
g_{1}(x)=\int_{0}^{\infty} r_{2}\left(\frac{x}{u}\right) g_{2}(u) \frac{d u}{u} .
$$

On replacing $u$ by $1 / u$ in (10); $x$ by $1 / x$ in (11); $g_{1}(1 / x)$ by $x h_{1}(x)$, $g_{2}(x)$ by $h_{2}(x) ; r_{1}(x)$ by $k_{1}(x)$ and $r_{2}(1 / x)$ by $x k_{2}(x)$ these equations become

$$
\begin{aligned}
& h_{2}(x)=\int_{0}^{\infty} k_{1}(x u) h_{1}(u) d u, \\
& h_{1}(x)=\int_{0}^{\infty} k_{2}(x u) h_{2}(u) d u .
\end{aligned}
$$

This form shows that (10) and (11) are essentially generalized Fourier transforms [2, Chap. 8; 4, Chap. 5].

The statement $g(x) \in L^{p}(a, b)$, or $g(x)$ lies in $L^{p}(a, b)$, means that $\int_{a}^{b}|g(x)|^{p} d x$ exists. When $p=1$ we write $L(a, b)$ instead of $L^{1}(a, b)$.

We shall prove two theorems, one when the functions lie in $L(0, \infty)$ and one when they lie in $L^{2}(0, \infty)$. The latter case, although more difficult, gives us much wider and more satisfactory conditions than does the former case. Theorem 2 contains as a special case the results of my previous paper on chain transforms [3].

3. The Mellin inversion formulae. The Mellin inversion formulae are given by

$$
\begin{aligned}
& R(s)=\int_{0}^{\infty} r(x) x^{s-1} d x, \\
& r(x)=\frac{1}{2 \pi i} \int_{c-i \infty}^{c+i \infty} R(s) x^{-s} d s .
\end{aligned}
$$

In this paper all pairs of functions written in the form $r(x), R(s)$; $l(x), L(s) ; g(x), G(s)$, etc. will be Mellin transforms of each other, as in (14), (15). We shall confine ourselves to the case when $c=1 / 2$ for the rest of this paper.

The properties of Mellin transforms needed for Theorem 1 are:

(A) (See $[2$, p. 48 , Theorem 28]). If

$$
y^{-1 / 2} g(y) \in L(0, \infty) ;
$$

(ii) $y^{-1 / 2} g(y)$ is of bounded variation in the neighbourhood of $y=x$ and

$$
G(s)=\int_{0}^{\infty} g(x) x^{s-1} d x,
$$


where $s=1 / 2+i t$ and $t$ is real, then

$$
\frac{1}{2}\{g(x+0)+g(x-0)\}=\frac{1}{2 \pi i} \lim _{T \rightarrow \infty} \int_{1 / 2-i T}^{1 / 2+i T} G(s) x^{-s} d s .
$$

(B) (See $[2$, p. 60, Theorem 44].) If

$$
\begin{gathered}
x^{-1 / 2} g(x) \in L(0, \infty) ; \\
x^{-1 / 2} r(x) \in L(0, \infty), \text { and } \\
h(x)=\int_{0}^{\infty} r\left(\frac{x}{u}\right) g(u) \frac{d u}{u},
\end{gathered}
$$

then $x^{-1 / 2} h(x) \in L(0, \infty)$ and the Mellin transform of $h(x)$ is $R(s) G(s)$ (i.e. $H(s)=R(s) G(s)$ ).

4. Theorem 1. If (i) $x^{-1 / 2} l_{q}(x) \in L(0, \infty)$ and $x^{-1 / 2} r_{p}(x) \in L(0, \infty)$ for all the kernels of (4) and (5); (ii)

$$
\prod_{q} L_{q}(s)=\prod_{p} R_{p}(s)
$$

where the product of the Mellin transforms of all the l-kernels appears on the left-hand side and of all the r-kernels on the right-hand side. If all the kernels are l-kernels then (16a) must be replaced by

$$
\prod_{q} L_{q}(s)=1 \text {, }
$$

and if all the kernels are r-kernels then (16a) is replaced by

$$
1=\prod_{p} R_{p}(s)
$$

(iii) $g_{1}(x)$ and a sufficient number of the functions $g_{i}(x)$ satisfy $x^{-1 / 2} g_{i}(x)$ $\in L(0, \infty)$ to enable us to apply (B), $\$ 3$ to all the equations of (4) and (5); and (iv) $y^{-1 / 2} g_{1}(y)$ is of bounded variation in the neighbourhood of $y=x$, then $g_{n+1}(x)=g_{1}(x)$ and the system (4), (5) is a chain transform of order $n$.

It will be seen later that condition (iii) frequently simplifies to the assumption that $x^{-1 / 2} g_{1}(x) \in L(0, \infty)$.

To prove the theorem, conditions (i) and (iii) enable us to apply (B), $\S 3$ to each of the equations of (4) and (5). We may then deduce that

$$
\begin{gathered}
G_{p+1}(s)=R_{p}(s) G_{p}(s), \\
L_{q}(s) G_{q+1}(s)=G_{q}(s),
\end{gathered}
$$

where $p$ and $q$, between them, run through all the integers from 1 to $n$. 
Every function $G_{2}(s), G_{3}(s), \cdots, G_{n}(s)$ occurs twice, once on each side of $(17),(18)$ and $G_{1}(s), G_{n+1}(s)$ occur once each, on opposite sides.

Now multiply all the equations of (17) and (18) together and use condition (ii). We obtain

$$
G_{1}(s)=G_{n+1}(s) .
$$

From condition (iv) and (A), $\S 3$ it then follows that $g_{1}(x)=g_{n+1}(x)$ and the proof is completed.

Condition (iii) simplifies when all the kernels are either all $r$-kernels or all $l$-kernels. If they are all $r$-kernels it is sufficient to assume that $x^{-1 / 2} g_{1}(x) \in L(0, \infty)$. For the chain transform equations are all of type (4) and none are of type (5). Hence, since $x^{-1 / 2} g_{1}(x)$ and $x^{-1 / 2} r_{1}(x)$ both belong to $L(0, \infty)$, we may apply (B), $\S 3$ to the first equation of (4) and deduce that $x^{-1 / 2} g_{2}(x) \in L(0, \infty)$. Since $x^{-1 / 2} r_{2}(x) \in L(0, \infty)$ we may deduce similarly from the second equation of (4) that $x^{-1 / 2} g_{3}(x) \in L(0, \infty)$. Evidently we may continue this process until we have proved that $x^{-1 / 2} g_{i}(x) \in L(0, \infty)$ for $i=2,3, \cdots,(n+1)$.

If all the kernels are $l$-kernels it is sufficient to assume that $x^{-1 / 2} g_{n+1}(x) \in L(0, \infty)$. Then, as above, we may deduce that this property is true for all the other $g_{i}(x)$ functions.

5. Examples of chain transforms according to Theorem 1. The cases $n=1$ and $n=2$ will be discussed in $\S 9$, after we have proved Theorem 2.

Equations (7), (8), and (9) provide us with an example of a chain transform of order 3 . We have $r_{1}(x)=(1 / x) e^{-1 / x} ; r_{2}(x)=e^{-x} ; l_{3}(x)$ $=1 /(1+x)$ and condition (i) is easily verified. The respective Mellin transforms are $R_{1}(s)=\Gamma(1-s) ; R_{2}(s)=\Gamma(s) ; L_{3}(s)=\pi / \sin (s \pi) \quad[2$, p. 192]. Thus condition (ii) is verified since $\Gamma(1-s) \Gamma(s)=\pi / \sin (s \pi)$. For (iii) it is sufficient to assume that $x^{-1 / 2} g_{1}(x) \in L(0, \infty)$ but we must then replace (iv) by $y^{-1 / 2} g_{3}(y)$ is of bounded variation in the neighbourhood of $y=x$.

To see this, from (7) we may deduce that $x^{-1 / 2} g_{2}(x) \in L(O, \infty)$ and then from (8) that $x^{-1 / 2} g_{3}(x) \in L(0, \infty)$. Now in (9) replace $g_{3}(x)$ by $g_{4}(x)$. By using the arguments of Theorem 1 we can prove that $G_{4}(s)$ $=G_{3}(s)$ and then deduce that $g_{4}(x)=g_{3}(x)$. This establishes (7), (8), and (9) as a chain transform of order 3.

In the case $n=3$ with two $r$-kernels and one $l$-kernel (16a) becomes

$$
L_{3}(s)=R_{1}(s) R_{2}(s) .
$$

From (B), $\S 3$ we may then infer that 


$$
l_{3}(x)=\int_{0}^{\infty} r_{1}\left(\frac{x}{u}\right) r_{2}(u) \frac{d u}{u} .
$$

When there are two $l$-kernels and one $r$-kernel the corresponding relationship is

$$
\int_{0}^{\infty} l_{2}\left(\frac{x}{u}\right) l_{3}(u) \frac{d u}{u}=r_{1}(x) .
$$

A further example of a chain transform of order 3 is given by the following kernels:

$$
\begin{gathered}
r_{1}(x)=2 x^{-\nu} J_{\nu}(2 x) ; \quad r_{2}(x)=e^{-1 / x} x^{-2 \nu-2} ; \\
l_{3}(x)=e^{-x^{2}},
\end{gathered}
$$

where $J_{\nu}(x)$ is the Bessel function of order $\nu$ [5, Chap. 3]. Condition (i) is satisfied if $\nu>0$. The respective Mellin transforms are [2, equation (7.9.1)],

$$
\begin{aligned}
& R_{1}(s)=\frac{\Gamma(s / 2)}{\Gamma(\nu-s / 2+1)} ; \quad R_{2}(s)=\frac{1}{2} \Gamma(\nu-s / 2+1) ; \\
& L_{3}(s)=\frac{1}{2} \Gamma(s / 2) .
\end{aligned}
$$

Condition (ii) is evidently satisfied. For (iii) it is sufficient to assume that $x^{-1 / 2} g_{1}(x) \in L(0, \infty)$ but (iv) must be replaced by $y^{-1 / 2} g_{3}(x)$ is of bounded variation in the neighbourhood of $y=x$.

6. Properties of Mellin transforms in the class $L^{2}$. We can obtain more general results when our functions belong to the class $L^{2}(0, \infty)$. The properties of Mellin transforms required in this case are as follows:

(C) $[2, \S 3.17]$. If $f(x) \in L^{2}(0, \infty)$ then

$$
\underset{a \rightarrow \infty}{\lim .} \int_{1 / a}^{a} f(x) x^{s-1} d x=F(s),
$$

where 1.i.m. denotes limit in the mean. Also $F(s) \in L^{2}(1 / 2-i \infty$, $1 / 2+i \infty)$ and

$$
\underset{a \rightarrow \infty}{\lim . m} \frac{1}{2 \pi i} \int_{1 / 2-i a}^{1 / 2+i a} F(s) x^{-s} d s=f(x) .
$$

Conversely if $F(s) \in L^{2}(1 / 2-i \infty, 1 / 2+i \infty)$, then (26) defines $f(x)$, which belongs to $L^{2}(0, \infty)$. 
If either $f(x) \in L^{2}(0, \infty)$ and $g(x) \in L^{2}(0, \infty)$ or $F(s) \in L^{2}(1 / 2-i \infty$, $1 / 2+i \infty)$ and $G(s) \in L^{2}(1 / 2-i \infty, 1 / 2+i \infty)$ then

$$
\int_{0}^{\infty} f(x) g(x) d x=\frac{1}{2 \pi i} \int_{1 / 2-i \infty}^{1 / 2+i \infty} F(s) G(1-s) d s .
$$

Consider now the function $f(y)=1$ when $0<y<x$ and $f(y)=0$ when $y>x$. Its Mellin transform is evidently $x^{s} / s$ which, as a function of $s$, is in $L^{2}(1 / 2-i \infty, 1 / 2+i \infty)$. Hence, if $R(s) \in L^{2}(1 / 2-i \infty, 1 / 2+i \infty)$, we have from (27)

$$
\int_{0}^{x} r(y) d y=\frac{1}{2 \pi i} \int_{1 / 2-i \infty}^{1 / 2+i \infty} R(s) \frac{x^{1-s}}{1-s} d s .
$$

We shall write

$$
\boldsymbol{r}(x)=\int_{0}^{x} r(y) d y
$$

and all pair of functions written in this way, $g(x), g(x) ; 1(x), l(x)$, etc., will be related as in (29). Thus, from (28), $r(x) / x$ and $R(s) /(1-s)$ are Mellin transforms of each other.

The assumption $R(s) \in L^{2}(1 / 2-i \infty, 1 / 2+i \infty)$ can now be very considerably relaxed. For if $R(s)$ is bounded on the line $s=1 / 2+i t$, for all real values of $t$, then $R(s) /(1-s) \in L^{2}(1 / 2-i \infty, 1 / 2+i \infty)$ and therefore, by (26), equation (28) is still true, although convergence is now replaced by convergence in mean square. If we can differentiate (28) with respect to $x$ through the integral sign, then (28) is equivalent to (15). But the assumption that $R(s)$ is bounded is not in itself sufficient justification for such differentiation. For example if $R(s)=1$ from (28) we obtain the step function $\boldsymbol{r}(x)=0$ when $0<x<1$ and $\boldsymbol{r}(x)=1$ when $x>1$. But in this case $r(x)$ cannot be obtained from the integral of (15) since this integral does not converge.

If $R(s)$ is bounded, whether $r(x)$ exists or not, we have $\boldsymbol{r}(x)$ defined by (28). On replacing $x$ by $u / x$ and $s$ by $1-s$ in (28) we see that $\boldsymbol{r}(u / x)$ and $R(1-s) u^{s} / s$ are Mellin transforms of each other and that both functions, the first as a function of $x$ and the second as a function of $s$, are in the class $L^{2}$. Hence, if $R(s)$ is bounded, $g(x) \in L^{2}(0, \infty)$ and

$$
\boldsymbol{h}(u)=\int_{0}^{\infty} \boldsymbol{r}\left(\frac{u}{x}\right) g(x) d x,
$$

we can deduce from (27) that 


$$
h(u)=\frac{1}{2 \pi i} \int_{1 / 2-i \infty}^{1 / 2+i \infty} \frac{R(s)}{1-s} G(s) y^{1-s} d s
$$

and then from (28) and (29) that

$$
H(s)=R(s) G(s) .
$$

Since $R(s)$ is bounded on $s=1 / 2+i t$ and $G(s) \in L^{2}(1 / 2-i \infty$, $1 / 2+i \infty)$, it follows that $H(s) \in L^{2}(1 / 2-i \infty, 1 / 2+i \infty)$ and so, from the converse of $(\mathrm{C}), \S 6$, we also have $h(x) \in L^{2}(0, \infty)$.

7. Statement and proof of Theorem 2. In the second theorem equations (4) and (5) are replaced by

$$
\begin{aligned}
& g_{p+1}(x)=\frac{d}{d x} \int_{0}^{\infty} \boldsymbol{r}_{p}\left(\frac{x}{u}\right) g_{p}(u) d u, \\
& \frac{d}{d x} \int_{0}^{\infty} 1_{q}\left(\frac{x}{u}\right) g_{q+1}(u) d u=g_{q}(x) .
\end{aligned}
$$

As in $\S 2, p$ and $q$, between them, run through the first $n$ positive integers. Each of the functions $g_{2}(x), g_{3}(x), \cdots, g_{n}(x)$ occurs twice in (33) and (34), once on each side, and the functions $g_{1}(x)$ and $g_{n+1}(x)$ occur once each, on opposite sides. The system is said to be a chain transform of order $n$ if $g_{n+1}(x)=g_{1}(x)$.

When differentiation through the integral signs, with respect to $x$, is permissible, (33) and (34) reduce to (4) and (5).

THEOREM 2. If (i) $L_{q}(s)$ and $R_{p}(s)$, the Mellin transforms of the kernels of (33) and (34), are all bounded when $s=1 / 2+i$ for all real values of $t$, (ii) these Mellin transforms satisfy one of the functional equations given in condition (ii) of Theorem 1 , and (iii) $g_{1}(x)$ and a sufficient number of the functions $g_{i}(x), i=2,3, \cdots,(n+1)$, belong to $L^{2}(0, \infty)$ to enable us to apply (27) to all the equations of (33) and (34), then $g_{n+1}(x)=g_{1}(x)$ almost everywhere and the system (33), (34) forms a chain transform of order $n$.

It will be seen later in $\$ 8$ that condition (iii) frequently simplifies to $g_{1}(x) \in L^{2}(0, \infty)$ or some equivalent statement.

To prove the theorem we note from (29) that (33) may be written in the form

$$
\boldsymbol{g}_{p+1}(x)=\int_{0}^{\infty} \boldsymbol{r}_{p}\left(\frac{x}{u}\right) g_{p}(u) d u .
$$

Conditions (i) and (iii) enable us to deduce that 


$$
G_{p+1}(s)=R_{p}(s) G_{p}(s)
$$

in the same way as (32) has been deduced from (30).

From (34) we have similarly

$$
L_{q}(s) G_{q+1}(s)=G_{q}(s) .
$$

In (36) and (37) each function $G_{2}(s), G_{3}(s), \cdots, G_{n}(s)$ occurs twice, once on each side, and $G_{1}(s)$ and $G_{n+1}(s)$ occur once each, on opposite sides. Hence, on multiplying all the equations of (36) and (37) together and using the functional relationship of condition (ii), it follows that

$$
G_{1}(s)=G_{n+1}(s) .
$$

But $g_{1}(x) \in L^{2}(0, \infty)$; hence $G_{1}(s)=G_{n+1}(s) \in L^{2}(1 / 2-i \infty, 1 / 2+i \infty)$ and so, from (26), $g_{1}(x)=g_{n+1}(x)$ almost everywhere. The system (33), (34) is therefore a chain transform of order $n$.

\section{Cases when the conditions can be simplified.}

(A) If the kernels are either all $r$-kernels or all $l$-kernels, then condition (iii) simplifies considerably. If they are all $r$-kernels, condition (iii) simplifies to the single assumption that $g_{1}(x) \in L^{2}(0, \infty)$.

To prove this statement we note that all the equations of the chain transform are of the type (33) and none of type (34). Consider now the first of these equations. Since $g_{1}(u)$ and $\boldsymbol{r}_{1}(x / u)$, as functions of $u$, both belong to $L^{2}(0, \infty)$ we can deduce that $g_{2}(x)$ also belongs to $L^{2}(0, \infty)$. The arguments are the same as those used in deducing from (30) that $h(x) \in L^{2}(0, \infty)$. Since $g_{2}(x)$ and $r_{2}(x / u)$ now both belong to $L^{2}(0, \infty)$ we can repeat the method to prove that $g_{3}(x)$ $\in L^{2}(0, \infty)$. We can then continue this process until we have proved that $g_{i}(x) \in L^{2}(0, \infty)$ for $i=2,3, \cdots,(n+1)$.

If all the kernels are $l$-kernels it is sufficient to assume that $g_{n+1}(x)$ $\in L^{2}(0, \infty)$ instead of condition (iii).

In my previous paper [3] chain transforms were defined only for the case when all the kernels are $r$-kernels. Thus the conditions outlined just above cover the results obtained in [3].

(B) Condition (iii) can be reduced to the single assumption $g_{1}(x)$ $\in L^{2}(0, \infty)$ if in addition to (i) we assume that on the line $s=1 / 2+i$, for all real values of $t$, either that $1 / L_{q}(s)$ is bounded for every $q$ or that $1 / R_{p}(s)$ is bounded for every $p$.

To prove this suppose that $1 / L_{q}(s)$ satisfies the requirement. If the chain transform equations (33), (34) commence with $p=1$, then the conditions $R_{1}(s)$ bounded on $s=1 / 2+i t$ and $g_{1}(x) \in L^{2}(0, \infty)$ enable us to infer that $g_{2}(x) \in L^{2}(0, \infty)$. The proof is given in the last part 
of $\S 6$. If the equations, however, commence with $q=1$, i.e. from (34)

$$
\frac{d}{d x} \int_{0}^{\infty} 1_{1}\left(\frac{x}{u}\right) g_{2}(u) d u=g_{1}(x)
$$

we cannot infer from $L_{1}(s)$ bounded on $s=1 / 2+i t$ and $g_{1}(x)$ $\in L^{2}(0, \infty)$ that $g_{2}(x) \in L^{2}(0, \infty)$. But the extra condition $1 / L_{1}(s)$ is bounded on $s=1 / 2+i t$ enables us to make this inference.

For if $M(s)=1 / L_{1}(s)$ we have $M(s)$ and $L_{1}(s)$ are bounded on $s=1 / 2+i t$ and $M(s) L_{1}(s)=1$. The first two conditions of Theorem 2 are therefore satisfied and we may construct a chain transform of order 2 with (38) as one of the equations and

$$
\frac{d}{d x} \int_{0}^{\infty} \boldsymbol{m}\left(\frac{x}{u}\right) g_{1}(u) d u=g_{2}(x)
$$

as the other. For condition (iii) we require either that $g_{1}(x)$ $\in L^{2}(0, \infty)$ or that $g_{2}(x) \in L^{2}(0, \infty)$. If $g_{1}(x) \in L^{2}(0, \infty)$ then, since $M(s)$ is bounded, we may infer from (39) by the method of $\S 6$ that $g_{2}(x) \in L^{2}(0, \infty)$ also. From this point and the relation $M(s) L_{1}(s)=1$ we can then establish the truth of (38) by the method of Theorem 2 . But the important result for us is the deduction from (39) that $g_{2}(x) \in L^{2}(0, \infty)$ also. To sum up, whether the system (33), (34) commences with $p=1$ or $q=1$, the extra condition together with $g_{1}(x) \in L^{2}(0, \infty)$ justifies the deduction that $g_{2}(x) \in L^{2}(0, \infty)$.

In a similar manner we can establish from the second equation of (33), (34) that $g_{3}(x) \in L^{2}(0, \infty)$ and so on successively until we prove that $g_{i}(x) \in L^{2}(0, \infty)$ for $i=2,3, \cdots,(n+1)$.

If $1 / R_{p}(s)$ is bounded for every $p$ we proceed in the same way to prove that (iii) reduces to the single assumption $g_{1}(x) \in L^{2}(0, \infty)$.

9. Examples of chain transforms according to Theorem 2. In the case $n=1$ we have only one equation and so only one kernel. Assuming that this is an $r$-kernel, from condition (ii) we have $R_{1}(s)=1$. Evidently condition (i) of Theorem 2 is satisfied. From (28)

$$
\boldsymbol{r}_{1}(x)=\frac{1}{2 \pi i} \int_{1 / 2-i \infty}^{1 / 2+i \infty} R_{1}(s) \frac{x^{1-s}}{1-s} d s=\left\{\begin{array}{rr}
0, & 0<x<1 \\
1, & x>1 .
\end{array}\right.
$$

From (33) the solitary chain transform equation becomes

$$
g_{1}(x)=\frac{d}{d x} \int_{0}^{x} g_{1}(u) d u=g_{1}(x) .
$$

Hence, from condition (iii), if $g_{1}(x) \in L^{2}(0, \infty)$, then the equation 
$g_{1}(x)=g_{1}(x)$ is a chain transform of order 1 .

When $n=2$ and both kernels are either $r$-kernels or $l$-kernels the chain transform reduces to the well known generalized Fourier transform [2, Chap. 8]. For example if we take

$$
\begin{aligned}
& R_{1}(s)=\Gamma(s) \cos (s \pi / 2) \\
& R_{2}(s)=\frac{2}{\pi} \Gamma(1-s) \sin (s \pi / 2),
\end{aligned}
$$

conditions (i) and (ii) of Theorem 2 are satisfied. The Mellin transforms $[2$, p. 196] are

$$
r_{1}(x)=\cos x ; \quad r_{2}(x)=\frac{2}{\pi x} \cos \left(\frac{1}{x}\right) .
$$

On writing out the two chain transform equations, which are both of type (33), differentiating through the integration sign, when justified, and making a few slight changes of variables this system reduces to the ordinary Fourier cosine transform.

Similarly, starting with $R_{1}(s)=\Gamma(s)$ sin $(s \pi / 2)$, we can reconstruct the Fourier sine transform.

Condition (ii), which in this case is either $R_{1}(s) R_{2}(s)=1$ or $L_{1}(s)$ $L_{2}(s)=1$, is equivalent to the functional equation discovered by Hardy and Titchmarsh $[6 ; 2,(8.3 .4)]$.

When $n=2$ and we have one $r$-kernel and one $l$-kernel condition (ii) becomes $L_{2}(s)=R_{1}(s)$. We then have $\boldsymbol{I}_{2}(x)=\boldsymbol{r}_{1}(x)$ and the chain transform equations, one of type (33) and the other of type (34), are seen to be repetitions of each other.

In my previous paper [3] I have given several examples of chain transforms of general order $n$ when all the kernels are either $r$-kernels or $l$-kernels. Many examples can be given when the kernels are of both types. I will give two new ones for the case $n=3$; they both come under Theorem 2 with condition (iii) as given in (B), §8. The first example is

$$
r_{1}(x)=\cos x ; \quad r_{2}(x)=\sin x ; \quad l_{3}(x)=\frac{\pi}{2} J_{0}\left(2 x^{1 / 2}\right),
$$

where $J_{0}(x)$ denotes the Bessel function of order zero [5, Chap. 3]. The corresponding Mellin transforms are [2, p. 196]

$$
\begin{aligned}
& R_{1}(s)=\Gamma(s) \cos (s \pi / 2) ; \quad R_{2}(s)=\Gamma(s) \sin (s \pi / 2) ; \\
& L_{3}(s)=\frac{\pi \Gamma(s)}{2 \Gamma(1-s)} .
\end{aligned}
$$


The second example is

$$
\begin{aligned}
& r_{1}(x)=2 \cos (2 \pi x) ; \quad r_{2}(x)=2 \cos (2 \pi x) ; \\
& l_{3}(x)=4 K_{0}\left(4 \pi x^{1 / 2}\right)-2 \pi Y_{0}\left(4 \pi x^{1 / 2}\right),
\end{aligned}
$$

where $K_{0}(x)$ and $Y_{0}(x)$ are functions associated with $J_{0}(x)[5, \mathrm{pp}$. $64,65]$. The corresponding Mellin transforms are [2, p. 220]

$$
\begin{aligned}
& R_{1}(s)=\frac{\zeta(1-s)}{\zeta(s)}=2^{1-s} \pi^{-s} \cos (s \pi / 2) \Gamma(s)=R_{2}(s) \\
& L_{3}(s)=\left\{\frac{\zeta(1-s)}{\zeta(s)}\right\}^{2},
\end{aligned}
$$

where $\zeta(s)$ is the Riemann zeta function. The $r_{1}(x)$ and $r_{2}(x)$ of (46) do not, according to chain transform theory, form the kernels of the Fourier cosine transform. The form they take when they are Fourier cosine kernels is given by (43).

\section{REFERENCES}

1. D. V. Widder, The Laplace transform, Princeton University Press.

2. E. C. Titchmarsh, Theory of Fourier integrals, Oxford University Press.

3. C. Fox, Chain transforms, J. London Math. Soc. vol. 23 (1948) pp. 229-235. A misprint occurs on p. 234 of this paper as follows: line 20, denominator of first integrand, for $\pi\left(1-x^{2}\right)$ read $\pi\left(x^{2}-u^{2}\right)$.

4. S. Bochner and K. Chandrasekharan, Fourier transforms, Annals of Mathematics Studies, no. 19.

5. G. N. Watson, Theory of Bessel functions, Cambridge University Press.

6. G. H. Hardy and E. C. Titchmarsh, $A$ class of Fourier kernels, Proc. London Math. Soc. (2) vol. 35 (1933) pp. 116-155.

MCGiLl University 